\title{
Enhancing cognition through PDE4 regulation
}

The cyclic nucleotide phosphodiesterase 4 (PDE4) regulates the hydrolysis of the second messenger cyclic AMP. cAMP is a major intracellular signalling mediator that directly affects the activity of cyclic-nucleotidedependent protein kinases and gated ion channels. PDE4 represents an attractive target for modulating neuronal activity and thus for treating psychiatric and neurodegenerative

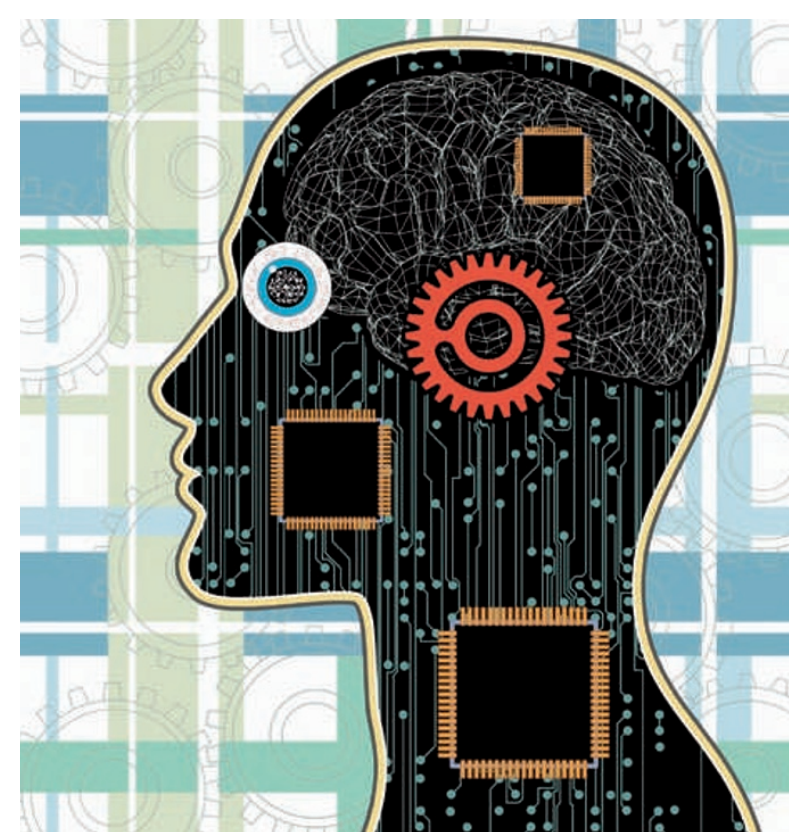

disorders. However, none of the PDE4 inhibitors tested so far has reached the market, mainly owing to tolerability issues such as emesis. A study in Nature Biotechnology that sheds light on the structural basis of PDE4D (a PDE4 isoform) regulation has led to the development of allosteric modulators with fewer side effects than existing compounds.

The existing PDE4 inhibitors that are directed at the active site are not isoform-specific and act as competitive inhibitors. Owing to complete inhibition of enzyme activity, the physiological levels of cAMP are likely to be altered, leading to side effects such as emesis and diarrhoea. In this study, Burgin et al. present crystal structures of inhibitor-bound PDE4, which show a previously unsuspected role for the regulatory domain UCR2 in controlling access to the enzyme's active site - UCR2 binds to both the inhibitors and the catalytic domain of PDE4. Subsequent structure-activity relationship studies of compounds that interact with UCR2 led to the identification of more than 800 compounds that do not completely inhibit PDE4 activity in vitro. In addition, mutagenesis studies showed that interactions with Phe196 in UCR2 are key for PDE4D selectivity.

Although such allosteric modulators only partially inhibit PDE4 activity, they are still able to block a biological response, as measured in an assay of leukotriene E4 production by eosinophils. Moreover, in rodent models of cholinergic deficit, in which memory consolidation is impaired, intravenous administration of four PDE4D allosteric modulators improved the animals' performance in a spatial working memory task (Y-maze) and long-term memory formation (assessed by the novel object recognition test) to a similar extent as the full PDE4 inhibitor rolipram. Importantly, the effect of these compounds on emesis was significantly reduced compared with rolipram, highlighting the potential of such compounds as cognitive enhancers for the treatment of Alzheimer's disease, Huntington's disease, schizophrenia and depression.

Monica Hoyos Flight

ORIGINAL RESEARCH PAPER Burgin, A. B. et al. Design of phosphodiesterase 4D (PDE4D) allosteric modulators for enhancing cognition with improved safety. Nature Biotech. 28, 63-70 (2009) 